\title{
AN ON-LINE SIGNAL-DEPENDENT TIME-FREQUENCY REPRESENTATION
}

\author{
Douglas L. Jones and Richard G. Baraniuk* \\ Coordinated Science Laboratory \\ University of Illinois \\ Urbana, IL 61801
}

\section{INTRODUCTION}

Time-frequency representations are a central signal analysis tool for a wide variety of applications. A large number of bilinear time-frequency distributions (TFD's) have been proposed, each differing only in the choice of a kernel function [1], [2]. In recent years, it has become apparent that no single kernel can give adequate performance for a large class of signals; hence, there has been increasing interest in signal-dependent or adaptive time-frequency representations, in which the kernel function varies with the signal to achieve nearoptimal performance for a large class of signals. Arguments for signal dependence and surveys of the related literature can be found in [2], [3].

A number of signal-dependent time-frequency representations have been developed. Among the most promising are those based on optimality criteria, including a signal-dependent time-frequency representation based on radially Gaussian kernels [4]. This representation performs well for a wide variety of signals, is fully automatic, and has a computational complexity of the same order as fixed-kernel methods. However, this block-oriented technique designs only one kernel for the entire signal. This limits the technique to processing only relatively short signals in an off-line fashion. Furthermore, this approach does not support adaptation of the kernel with time, which has been shown to be beneficial in many situations [3]. A need exists for a computationally efficient, time-adaptive, signal-dependent time-frequency representation suitable for on-line operation.

\section{RADIALLY GAUSSIAN KERNELS}

The signal-dependent TFD proposed in [4] is based on kernels with Gaussian radial cross sections. In polar coordinates, a radially Gaussian kernel is expressed as

$$
\Phi(r, \psi)=e^{-\frac{r^{2}}{2 \sigma^{2}(\psi)}}
$$

where $r$ and $\psi$ represent radius and angle, respectively. The function $\sigma(\psi)$ controls the spread of the kernel along a radial slice at angle $\psi$; we call it the spread function. Clearly, if $\sigma$ is smooth, then $\Phi$ is also smooth.

A high quality time-frequency representation results when the kernel is well matched to the components of a given signal. The radially Gaussian kernel is adapted to

\footnotetext{
*Sponsored by the National Science Foundation, Grant No. MIP 90-12747, the Joint Services Electronics Program, Grant No. No0014-90-J-1270, and the Computer-based Education Research Laboratory.
}

a signal by solving the following optimization problem:

$$
\max _{\Phi} \int_{0}^{2 \pi} \int_{0}^{\infty}|A(r, \psi) \Phi(r, \psi)|^{2} r d r d \psi
$$

subject to

$$
\begin{gathered}
\Phi(r, \psi)=e^{-\frac{r^{2}}{2 \sigma^{2}(\psi)}} \\
\frac{1}{2 \pi} \int_{0}^{2 \pi} \int_{0}^{\infty}|\Phi(r, \psi)|^{2} r d r d \psi \leq \alpha, \quad \alpha \geq 0 .
\end{gathered}
$$

Here, $A(r, \psi)$ is the ambiguity function (AF) of the signal in polar coordinates. The AF is utilized in the optimization formulation because of its important separation property: the auto and cross-components separate somewhat in the plane, with the auto-components lying centered at the origin and the cross-components lying away from the origin [5].

The constraints and performance index are motivated by a desire to suppress cross-components and to pass auto-components with as little distortion as possible. The first constraint (3) limits the scope of the optimization to the class of radially-Gaussian kernels. Thus, the kernel is constrained to be a lowpass filter. Since the AF auto-components are centered at the origin, this encourages the kernel to preferentially pass auto-components. The second constraint (4) limits the volume of the kernel to $\alpha$, so that cross-components are suppressed. The performance measure (2) expresses a desire to minimize auto-component distortion by passing as much autocomponent energy as possible into the TFD for a kernel of fixed volume. An advantage of this formulation is that the constraints are insensitive to both the timescale and orientation of the signal in time-frequency.

The shape of the kernel is completely parameterized by the one-dimensional spread function $\sigma$, so finding the optimal, radially-Gaussian kernel $\Phi_{\text {opt }}$ for a signal is equivalent to finding the optimal function $\sigma_{\text {opt }}$ for the signal. A gradient-projection procedure efficiently solves the above optimization problem in $O\left(I M^{2}\right)$ computations, where $M$ is the number of frequency samples in the TFD and $I$ is the nurnber of iterations (typically less than 30) [4]. The optimal-kernel TFD is computed as the 2-d Fourier transform of the product of the AF and the optimal kernel.

\section{AN ON-LINE ALGORITHM}

We now describe a time-adaptive version of the above technique. The key to the algorithm is a "short-time ambiguity function" (STAF), which we define as

$$
A_{t}(\theta, \tau)=\int_{t-\tau_{1}}^{t+\tau_{1}} s^{*}\left(u-\frac{\tau}{2}\right) s\left(u+\frac{\tau}{2}\right) e^{j \theta u} d u .
$$


The STAF differs from the usual AF only in the limits of integration. The algorithm proceeds as follows. At each time $t$, the optimization in (2)-(4) is solved for the optimal kernel using a polar coordinate version of the STAF in place of the global AF, and a single constanttime slice of the TFD is computed as one slice of the 2-d Fourier transform of the rectangular coordinate STAFkernel product. Since the STAF varies with time, so does the optimal signal-dependent kernel.

Recursive computation of the STAF greatly reduces the computational burden of this technique. For an appropriate choice of $\tau_{1}$, the STAF can be rewritten in a "causal" form via the change of variable $v=u+\tau / 2$

$$
A_{t}(\theta, \tau)=e^{-j \theta \tau / 2} \int_{t-\tau_{2}}^{t} s(v) s^{*}(v-\tau) e^{j \theta v} d v
$$

Discretizing, this becomes

$$
\begin{aligned}
& A_{n}(m, k)=e^{-j \theta_{m} \tau_{k} / 2} . \\
& \quad \sum_{p=0}^{P_{m, k}-1} s(T n-T p) s^{*}\left(T n-T p-\tau_{k}\right) e^{j \theta_{m} T p}
\end{aligned}
$$

The sum in (7) can be efficiently computed using the recursion

$$
\begin{gathered}
y_{n}(m, k)=e^{j \theta_{m} T} y(n-1)+s(n) s^{*}\left(n-\tau_{k}\right) \\
-e^{j \theta_{m} T P_{m, k}} s\left(n-P_{m, k}\right) s^{*}\left(n-P_{m, k}-\tau_{k}\right) .
\end{gathered}
$$

Both the polar and rectangular coordinate STAF's can be efficiently computed in this manner, at a constant cost for each STAF sample. The total cost to update the $M^{2}$ samples of the polar and rectangular STAF's is thus $O\left(M^{2}\right)$.

Optimization of the radially Gaussian kernel proceeds according to the gradient-project algorithm developed in [4]. Since the STAF changes only slightly from one time sample to the next, only one or two gradient steps need be taken each time increment to track the optimal kernel over time, at a cost of $O\left(M^{2}\right)$ operations.

Finally, the current-time slice of the time-frequency representation is computed as the 2-d Fourier transform of the product of the rectangular STAF and the kernel at the output time $n-n_{0}$. For a single time, this computation requires $M$ row accumulations (cost $O\left(M^{2}\right)$ ) and a single column FFT.

\section{EXAMPLE}

Figure 1 presents a contour plot of a short-time Fourier transform (STFT) of a test signal containing a variety of different components. Amplitude variations and excessive broadening are clearly visible in portions of the STFT. Figure 2 contains the adaptive TFD of this signal computed using the algorithm described above. This representation is much more concentrated than the STFT and exhibits much less amplitude distortion.

\section{CONCLUSION}

Signal-dependent time-frequency representations offer good performance for a large class of signals. By employing adaptation over time of the kernel, the algorithm developed here tracks time variations in a signal. Certain constraints, such as outer time support (cone constraints), can easily be incorporated into the algorithm at no additional cost. The proposed implementation supports efficient, on-line adaptation of the kernel and running computation of the time-frequency representation, thus extending the benefits of adaptive time-frequency representation to real-time applications and signals of arbitrary length. The computational cost of this method is $O\left(N M^{2}\right)$, where $N$ is the signal length in samples and $M$ is the number of frequency samples produced at each time. This exceeds the $O(N M \log M)$ cost of most fixed-kernel representations, but the benefits of adaptivity may often outweigh the additional cost.

\section{REFERENCES}

1. L. Cohen, "Time-Frequency Distributions - A Review," Proceedings of the IEEE 77(7), pp. 941981, July 1989.

2. F. Hlawatsch and G.F. Boudreaux-Bartels, "Linear and Quadratic Time-Frequency Signal Representations," IEEE Signal Processing Magazine, Vol. 9 No. 2, pp. 21-67, April, 1992.

3. D.L. Jones and T.W. Parks, "A High Resolution Data-Adaptive Time-Frequency Representation," IEEE Trans. Acoust., Speech, and Signal Processing, Vol. 38, No. 12, December 1990, pp. 2127-2135.

4. R.G. Baraniuk and D.L. Jones, "A Radially Gaussian Signal-Dependent Time-Frequency Representation," IEEE ICASSP-91, pp. 3181-3184.

5. P. Flandrin, "Some features of Time-Frequency Representations of Multicomponent Signals," IEEE ICASSP-84, pp. 41.B.4.1-41.B.4.4.

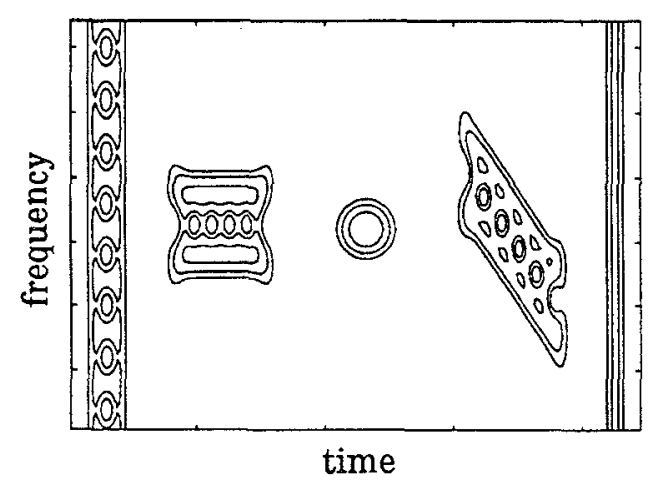

Figure 1: STFT of a multi-component signal.

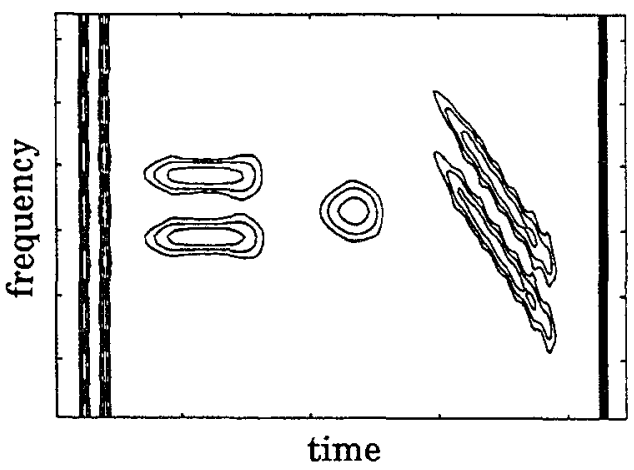

Figure 2: On-line optimal radially Gaussian kernel TFD of a multi-component signal. 